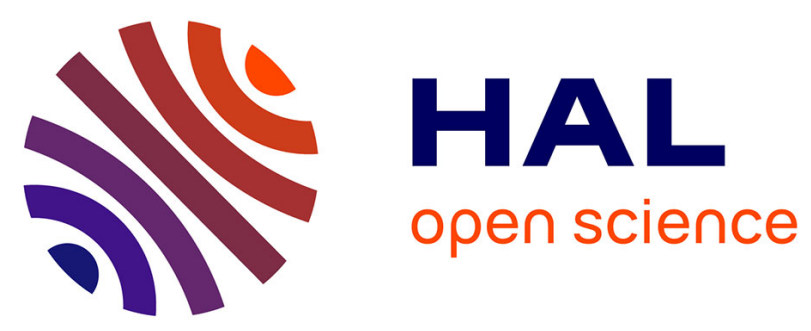

\title{
Comparison of various CFD codes for les simulations of turbomachinery: from inviscid vortex convection to multi-stage compressor
}

Vincent Brunet, Emma Croner, Alexandre Minot, Jérôme de Laborderie, Eric Lippinois, Stéphane Richard, Jean-François Boussuge, Jérôme Dombard, Florent Duchaine, Laurent Gicquel, et al.

\section{To cite this version:}

Vincent Brunet, Emma Croner, Alexandre Minot, Jérôme de Laborderie, Eric Lippinois, et al.. Comparison of various CFD codes for les simulations of turbomachinery: from inviscid vortex convection to multi-stage compressor. ASME Turbo Expo, Jun 2018, Oslo, Norway. 10.1115/GT2018-75523 . hal-02003192

\section{HAL Id: hal-02003192 \\ https://hal.science/hal-02003192}

Submitted on 13 Feb 2019

HAL is a multi-disciplinary open access archive for the deposit and dissemination of scientific research documents, whether they are published or not. The documents may come from teaching and research institutions in France or abroad, or from public or private research centers.
L'archive ouverte pluridisciplinaire HAL, est destinée au dépôt et à la diffusion de documents scientifiques de niveau recherche, publiés ou non, émanant des établissements d'enseignement et de recherche français ou étrangers, des laboratoires publics ou privés. 


\section{COMPARISON OF VARIOUS CFD CODES FOR LES SIMULATIONS OF TURBOMACHINERY: FROM INVISCID VORTEX CONVECTION TO MULTI-STAGE COMPRESSOR}

\author{
Vincent Brunet, Emma Croner, \\ Alexandre Minot \\ Safran Tech \\ Chateaufort, France \\ Jean-François Boussuge, \\ Jérôme Dombard, Florent \\ Duchaine, Laurent Gicquel, \\ Thierry Poinsot, Guillaume \\ Puigt, Gabriel Staffelbach, \\ Luis Segui, Olivier Vermorel, \\ Nadège Villedieu \\ Cerfacs \\ Toulouse, France
}

\author{
Jérôme de Laborderie, Eric \\ Lippinois \\ Safran Aircraft Engines \\ Villaroche, France
}

\author{
Jean-Sébastien Cagnone, \\ Koen Hillewaert, Michel \\ Rasquin \\ Cenaero \\ Charleroi, Belgium
}

\author{
Stéphane Richard \\ Safran Helicopter Engines \\ Bordes, France
}
Ghislain Lartigue, Vincent Moureau

CNRS / CORIA

Rouen, France

\author{
Vincent Couailler, Emeric \\ Martin, Marta de la Llave Plata, \\ Jean-Marie Le Gouez, Florent \\ Renac \\ Onera \\ Châtillon, France
}

\begin{abstract}
Some possible future High Fidelity CFD codes for LES simulation of turbomachinery are compared on several test cases increasing in complexity, starting from a very simple inviscid Vortex Convection to a multistage axial experimental compressor. Simulations were performed between 2013 and 2016 by major Safran partners (Cenaero, Cerfacs, CORIA and Onera) and various numerical methods compared: Finite Volume, Discontinuous Galerkin, Spectral Differences. Comparison to analytical results, to experimental data or to RANS simulations are performed to check and measure accuracy. CPU efficiency versus accuracy are also presented. It clearly appears that the level of maturity could be different between codes and numerical approaches. In the end, advantages and disadvantages of every codes obtained during this project are presented.
\end{abstract}

\begin{tabular}{ll}
\multicolumn{2}{c}{ NOMENCLATURE } \\
DoFs & $\begin{array}{l}\text { Degrees Of Freedom } \\
\text { polynomial points for HO) }\end{array}$ \\
FV & Finite Volume \\
HO & High-Order \\
IGV & Inlet Guiding Vane \\
LES & Large Eddy Simulation \\
$\mathrm{M}$ & Mach number \\
$\mathrm{P}$ & Pressure \\
$\mathrm{p}$ & Polynomial order for HO codes \\
$\mathrm{RANS}$ & Reynolds Averaged Navier-Stokes \\
$\mathrm{Re}$ & Reynolds number \\
$\mathrm{T}$ & Temperature \\
$\mathrm{Tu}$ & Turbulence intensity \\
$\delta$ & Boundary layer thickness
\end{tabular}

\section{INTRODUCTION}

Turbomachinery simulation clearly benefit from unsteady approaches in terms of accuracy [17],[32],[33]. Rotor / stator interactions are indeed unsteady phenomena that have to be 
computed at least with a unsteady RANS approach [17],[32] because of the periodic wakes that impact downstream rows. Furthermore, complex phenomena into turbomachinery like separations, laminar-to-turbulent transition or leakage flows may not be properly captured with a turbulence model, and so LES (or hybrid RANS-LES) may be required for better accuracy [17],[33]. This future trend is also described into the well-known "NASA-2030” report [30].

In 1997, Spalart et al [31] predicted that many years will still be necessary before the first resolved and accurate LES of an aircraft wing will be completed. Nevertheless, considering LES, Re number is an important parameter for CPU cost and this number is significantly smaller for turbomachinery compared to aircraft wings. Note that it can strongly vary between the fan and a low pressure turbine. According to revisited LES cost as a function of Re number of Choi and Moin [8] (from Chapman [7]), LES can now be accessible for some turbomachinery, especially if we consider Wall-Modelled LES i.e. with wall functions.

The constant increase in computer capabilities now enables Large Eddy Simulations to be performed on full compressor configurations. Nonetheless, special attention to CPU cost and numerical scheme accuracy should be given. Many opened questions still remain on the future necessary LES numerical methods, spatial schemes, temporal integration, robustness etc. At present time, LES simulations have to be carried out by specialists, and many checks have to be performed before the exploitation of results. Hence, Safran has conducted a project called CN2020 ("Cours Numériques 2020”) with its major research partners to benchmark various LES oriented codes:

- TurboAVBP from Cerfacs: Unstructured Explicit Finite Volume / Finite Element code

- ARGO from Cenaero: Unstructured Implicit HighOrder Discontinuous Galerkin code

- Jaguar from Cerfacs: Unstructured Explicit HighOrder Spectral Difference code

- YALES2 from CORIA: Unstructured Implicit Explicit Finite Volume code

- Aghora from Onera: Unstructured Implicit High-Order Discontinuous Galerkin code

The project started in 2013 and was completed in the end of 2016. The objective was to progressively increase the test case complexity to demonstrate basic numerical accuracy and associated cost from very fundamental cases to turbomachinery applications. The four test cases are:

- Inviscid vortex convection with and without a sliding block: Comparisons of numerical accuracy on various grids and CPU costs for a given accuracy.
- Turbulent channel flow with heat transfer: First basic turbulent viscous case.

- $\quad$ LS89 VKI Turbine blade: Heat Transfer / Transition / Upstream turbulent effect / Numerical shock robustness.

- 3.5 Compressor Stage including one stator cavity: Demonstration on a realistic compressor with experimental reference.

All cases were computed but not by all partners because of the growing difficulty of the simulations. In particular the results obtained by Aghora, which was at the first stages of its development, correspond to the status of the code in 2014, knowing that the updated capacities of the code are described for instance in [25],[11],[26],[12]. Accurate comparisons with measurements or theory are carried out at least on the first three cases. For the two applied configurations, the LS89 and Create compressor, "classical" RANS simulations were also carried out to provide a reference.

The objective is to provide a state of the art of the different approaches that will be used for future compressor designs. Among all the objectives of this project, the main one aims at the evaluation of the necessary investments for the future in order to obtain a robust and accurate CFD high-fidelity LES method for a given CPU cost. If "classical" FV CFD software has already reached a high level of maturity, we also have to consider the potential of $\mathrm{HO}$ methods like Discontinuous Galerkin or Spectral Differences.

Please note that presented results correspond to the ones presented during the project and may be not fully representative of present capabilities of tested CFD codes (For example, see references for new CFD code capabilities).

\section{TEST CASES}

\section{Inviscid Vortex Convection}

The objective of the first test case was to compute the convection of a vortex in an inviscid flow. First, these simulations were performed on a classical single mesh with full periodicity as presented in [35]. Secondly, in order to prepare for turbomachinery applications, the computed domain was split into three regions: 2 being fixed and the middle one in translation as presented in Figure 1 in order to mimic stator/rotor/stator configurations. Every border of the domain is considered as periodic. Three translation velocities were considered for the middle domain $\left(V_{\text {Trans }}=0 / 50 / 300 \mathrm{~m} / \mathrm{s}\right)$, the highest one being representative of the velocity speed of a modern compressor close to the rotor tip. 


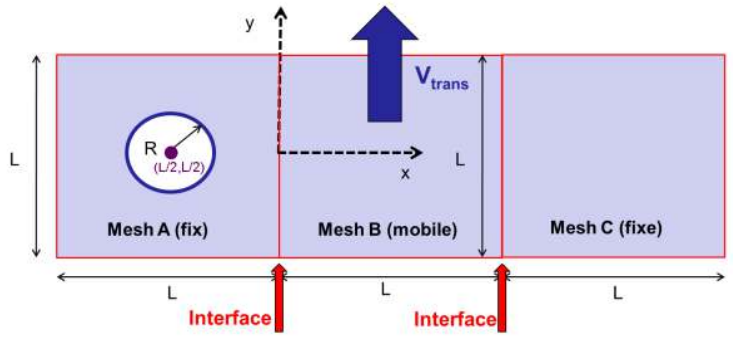

Figure 1 - Description of the Inviscid Vortex Convection case

Meshes with various densities, qualities and element types were provided and shared between partners:

- Regular quads (4 mesh densities and $M=0.05 /$ $0.5)$

- Unstructured quads (4 mesh densities $-M=0.01 /$ $0.05 / 0.5)$

- Unstructured triangles (4 mesh densities $-M=$ $0.05 / 0.5)$

This test case was computed by all CFD codes of partners with shared grids. The main objective was to validate the accuracy of CFD codes including the sliding block and to compare the ratio Accuracy/CPU costs in order to determine, for a given accuracy the less CPU expensive code. In this paper, only results obtained at $M=0.5$ are presented.

\section{Turbulent plane channel with imposed wall temperature}

The selected test case, from Abe et al [1] is a fully developed turbulent plane channel flow between two parallel and uniform heated walls, separated by $2 \delta$. The Reynolds number, based on the mean velocity $u_{b}$ is equal to $\operatorname{Re}_{b}=\frac{u_{b} 2 \delta}{v}$ and $M=0.1$ meaning that the flow is almost incompressible.

The spatial domain, represented in Figure 2, corresponds to $L x=12.8 \delta, L y=2 \delta, L z=6.4 \delta$ with a spatial resolution of 256 x 64 x 256 (roughly $4.2 \mathrm{M}$ DoFs). The local dimensionless cell sizes thus correspond to $\Delta x+\approx 32, \Delta y+\approx 0.6 \ldots 32$, $\Delta z+\approx 16$.

This test case was computed by all CFD codes of partners and the main objective was to evaluate the turbulent viscous capabilities and to compare CPU costs.

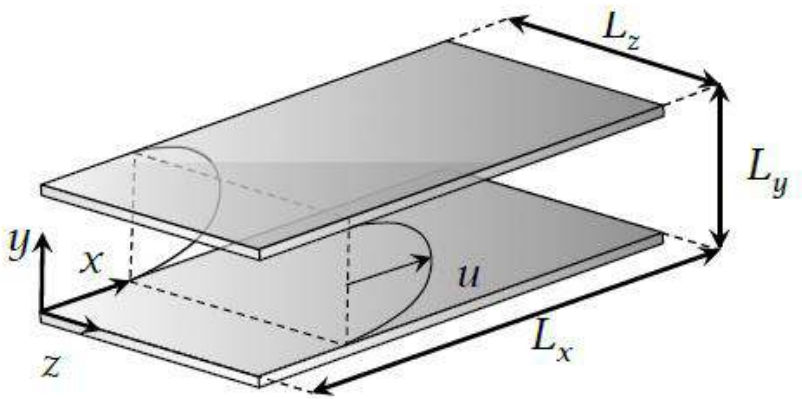

Figure 2 - Description of the turbulent periodic plane channel case

\section{LS89 Turbine blade cascade from VKI}

The LS89 Turbine blade cascade, tested at VKI [2] is a cooled-turbine cascade configuration. It was tested and widely computed and many configurations with various incoming turbulent intensity or outlet pressure exist.

Note that the original geometry description has to be slightly modified to prevent numerical oscillations on the suction side. The considered geometry retained for this study was imposed to all partners of the present project.

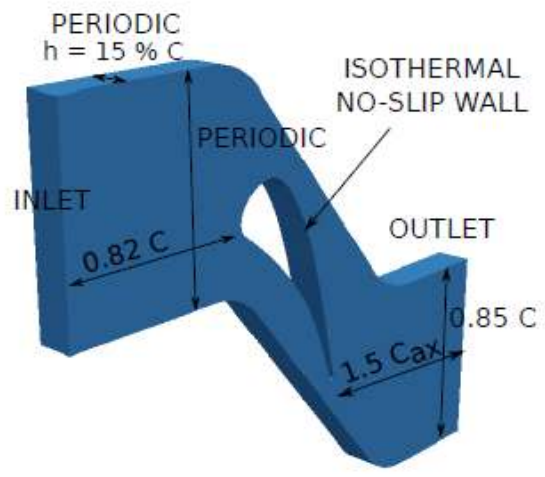

Figure 3 - LS89 Turbine Blade cascade simulation domain (from [28])

Among all measured test cases, three of them were computed as presented in Table 1.

\begin{tabular}{|cccc|} 
& MUR235 & MUR129 & HS \\
\hline $\mathbf{P}_{\mathbf{0 1}}(\mathbf{b a r})$ & 1.828 & 1.849 & 1.828 \\
$\mathbf{T}_{\mathbf{0 1}}(\mathbf{K})$ & 413.3 & 409.2 & 413.3 \\
$\mathbf{P}_{\mathbf{s} 2} / \mathbf{P}_{\mathbf{0 1}}$ & 0.57 & 0.63 & 0.3604 \\
$\mathbf{T}_{\mathbf{w}}$ & 301.15 & 297.75 & 301.15 \\
$\mathbf{M}$ at outlet & 0.927 & 0.84 & \\
Re at outlet & $1.2510^{6}$ & $1.135210^{6}$ & \\
Inlet Tu (\%) & 6 & 1 & 0 \\
\hline
\end{tabular}

Table 1 - Operating conditions for the LS89 test case

Note that the LS89 HS test case was not measured during the experimental campaign in VKI but was created numerically 
in order to test CFD codes with a strong shock / boundary layer interaction.

This test case was computed by ARGO, TurboAVBP, Jaguar and Yales 2 codes of the partners and the main objective was to evaluate the capabilities of these codes to accurately simulate this flow and evaluate their robustness with a strong shock / boundary layer interaction. Based on that, even if many exchanges between partners were performed, they were free to generate their own meshes and their own turbulent synthetic generation processes.

\subsection{Multistage CREATE2Bis compressor}

The experimental axial multistage CREATE2Bis compressor was designed by Safran Aircraft Engines and operated in LMFA (Ecole Central de Lyon, France). This compressor is representative of a modern compressor and is made of three axial stages + IGV as presented in Figure 4.

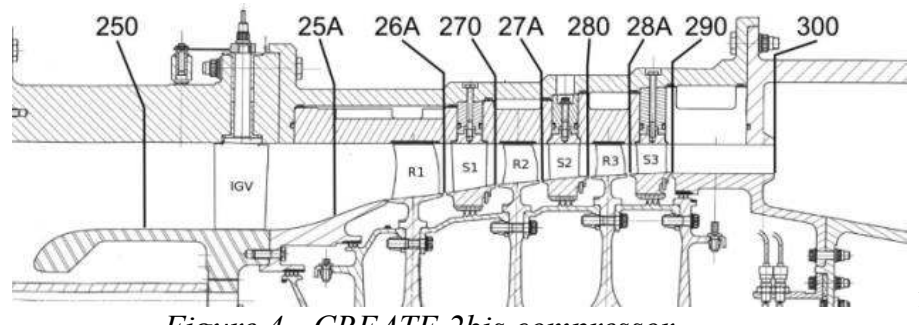

Figure 4 - CREATE 2bis compressor

It was designed so that vane and blade numbers are adapted to a natural periodicity of $2 \pi / 16$ to ensure a reduction of the modeling domain. These characteristics are presented in Table 2. To ensure a representative simulation, the entire compressor from plane 250 to plane 300 , including IGV blades, has to be computed. Inlet profile in plane 250 is provided from measurements. Furthermore, to increase the numerical and mesh complexity, partners have to simulate the cavity and labyrinth seal under the first stator as well as all the rotor radial tip clearances. The domain to be computed is presented in Figure 5 .

\begin{tabular}{|lccccccc|}
\hline Row & IGV & R1 & S1 & R2 & S2 & R3 & S3 \\
\hline $\mathbf{2} \boldsymbol{\pi}$ & 32 & 64 & 96 & 80 & 112 & 80 & 128 \\
$\mathbf{2 \pi / 1 6}$ & 2 & 4 & 6 & 5 & 7 & 5 & 8 \\
\hline
\end{tabular}

Table 2 - Blade numbers for each row of the CREATE2bis

compressor

This test case had to be computed by ARGO and TurboAVBP, these objectives being to evaluate the capabilities of these codes to perform this simulation with a minimum of 1 billion DoFs and a demonstration of 50000 CPU cores. Even with this large amount of DoFs, criteria to reach a Wall Resolved LES are not fulfilled. Hence simulations have to be carried out with wall functions.
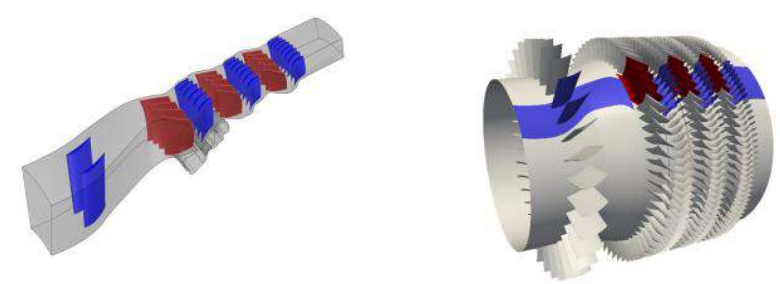

Figure 5-CFD Domain to be computed for the CREATE2bis Compressor [13]

A single operating point of the compressor is targeted for the simulation, corresponding to the nominal point at a rotation speed of $11543 \mathrm{rpm}$ and a target mass flow of $12.0 \mathrm{~kg} / \mathrm{s}$.

Note that this capability was not demonstrated with the ARGO code because of the non-availability of the CPU resources. On the contrary, Cerfacs performed the required simulation as well as several other operating points, but with coarser grids.

\section{CFD CODE DESCRIPTIONS}

\section{Cenaero - ARGO}

ARGO is an object-oriented $\mathrm{C}++$ high-order multi-physics solver based on the discontinuous Galerkin method (DG). DG finite-elements consider piecewise basis functions, which are discontinuous across element boundaries. Coupling between neighboring elements is limited to interface fluxes, thereby enabling arbitrarily high-order accuracy on irregular unstructured meshes. This compact footprint also offers high computational density and data locality, leading to excellent serial and parallel performance [18]. Transitional and turbulent flows are tackled with the so-called implicit LES strategy, where numerical dissipation plays the role of the subgrid scale model [4],[5]. The code was recently validated for shockturbulence interaction [19], and extended to Wall-Modeled LES [16].

\section{Cerfacs - Jaguar}

Jaguar [6] is a Fortran 90 high-order (up to tenth-order) solver designed to solve the Navier-Stokes equations following a Spectral Difference (SD) approach. The SD method is a member of spectral discontinuous techniques [34] and the key points are a polynomial reconstruction of variables inside any mesh elements and a Riemann solver to take into account explicitly discontinuity at mesh interface. The principle of SD is to solve the Navier-Stokes equations in their strong form, avoiding any integral formalism. Time integration is done using an explicit six step Runge-Kutta technique; turbulence modeling can be done using an implicit (via numerical dissipation) or explicit (Smagorinsky or WALE) LES model. Concerning the HPC efficiency, Jaguar was successfully tested up to 130000 cores with a flat MPI paradigm. 


\section{Cerfacs - TurboAVBP}

AVBP is an unstructured cell-vertex Finite Volume/Finite Element LES flow solver [27] co-developed by CERFACS and IFP-EN that resolves the laminar and turbulent compressible Navier-Stokes equations in two or three dimensions. It is capable of handling hybrid grids and different cell types (tetrahedra, hexahedra, prisms, etc). This effort is motivated by the efficiency of unstructured grid generation, the accuracy of the computational results (using regular structured elements) and the ease of mesh adaptation for industrial flow applications. AVBP focuses on unsteady turbulent flows (with and without chemical reactions) for internal flow configurations. The basic numerical methods are based on a Lax-Wendroff (LW) and a finite-element type low-dissipation Taylor-Galerkin (TGCC) scheme [10]. AVBP libraries include integrated parallel domain partitioning and data reordering tools, handles message passing and includes supporting routines for dynamic memory allocation, parallel input/output $(\mathrm{I} / \mathrm{O})$ and iterative methods.

TurboAVBP is a code developed at CERFACS for the simulation of turbomachinery flows by coupling multi-copies of a massively parallel unstructured compressible LES solver AVBP with the parallel coupler OpenPALM [15]. In the proposed strategy termed MISCOG, the rotor/stator interface is dealt with a coupling method based on the overset grid approach. The coupling method is proven to handle acoustic and vortical wave propagation for both interfaces with an acceptable degree of accuracy for LES. This is guaranteed provided the overlapping region contains a sufficiently large number of points dependent on the selected numerical scheme and on the order of interpolation [36].

\section{CORIA - YALES2}

YALES2 is a low-Mach number code capable of handling large unstructured meshes on massively parallel systems. It solves the unsteady filtered Navier-Stokes equations with the projection method of Chorin [9] adapted by Kim \& Moin [20] to variable density flows. These projection methods enable to remove the constraints due to acoustics at low-Mach number. For compressible flows, the code relies on a pressure-based prediction-correction time integration based on the characteristic splitting from Moureau et al. [24]. The space integration is made with a 4th-order central scheme and the time integration relies on a 4th-order scheme, which combines a 4thorder Runge-Kutta scheme with a Lax-Wendroff-like scheme also of the 4th-order. The solving of the linear systems that arise in the projection methods is performed with a Deflated Preconditioned Conjugate Gradient developed by Malandain et al. [23].

\section{Onera - AGHORA (\& NXO)}

Aghora is a DG prototype software developed for the numerical simulation of turbulent flows including the different levels of turbulence modeling, DNS, LES, RANS and hybrid RANS/LES [25],[11],[26][12]. The modal DG method has been initially developed and the nodal method has been added later on. At the time of the test case computations only the modal approach was available. It is that proposed in by Bassi and Rebay and relies on the use of a hierarchy of orthogonal polynomial functions as basis for the Galerkin projection. The discrete orthogonal polynomial spaces are directly computed in physical space. The methodology consists of defining a starting set of monomial basis functions in each (arbitrarily shaped) element and applying a modified Gram-Schmidt orthogonalization procedure. This ensures the orthogonality of the basis, and thereby the diagonal aspect of the mass matrix. Numerical integration on general-shaped elements is performed by means of a Gaussian quadrature. Space discretization use either the LLF or the Roe schemes for the convective fluxes and either the BR2 or the SIP schemes for the viscous fluxes. LLF and $\mathrm{BR} 2$ have been used in the present paper.

The time integration is performed using either explicit Runge-Kutta method up to 4th order accurate or implicit schemes with various integration techniques [25]. The test cases performed in 2014 have been run with the explicit techniques. Since 2013 MPI parallel efficiency has been regularly assessed within the context of several GENCI projects and PRACE Preparatory projects up to 22,000 cores on Tier-0 supercomputers.

The NXO software [21],[22] is based on high-order space interpolation from volume-averaged to surface-averaged quantities. In the FV context of collocated, cell centered methods, the reconstruction algorithm can be expressed synthetically in the following manner: Given a finite span of polynomial test functions in the space coordinates, extending over a sufficiently wide stencil of cells in the vicinity of the interface, and an arbitrary space function derive the set of linear interpolation coefficients $\mathrm{k}$ relating the known volume-averaged discrete field of values on the cells comprising the stencil $\mathrm{C}$ to a surface-averaged value of this field on a given interface. The consistent linear interpolation coefficients for the surfaceaveraged components of the gradient of the field on this same interface can also be defined in an analogous way.

\section{SUMMARY}

The Table 3 sum up the matching between CFD codes and test cases.

\begin{tabular}{|c|c|c|c|c|}
\hline & $\begin{array}{c}\text { Vortex } \\
\text { Convection }\end{array}$ & $\begin{array}{c}\text { Turbulent } \\
\text { plane } \\
\text { channel }\end{array}$ & LS89 & $\begin{array}{c}\text { CREATE } \\
\text { 2bis }\end{array}$ \\
\hline ARGO & $\mathrm{X}$ & $\mathrm{X}$ & $\mathrm{X}$ & $\mathrm{X}$ \\
\hline Jaguar & $\mathrm{X}$ & $\mathrm{X}$ & $\mathrm{X}$ & \\
\hline TurboAVBP & $\mathrm{X}$ & $\mathrm{X}$ & $\mathrm{X}$ & $\mathrm{X}$ \\
\hline YALES2 & $\mathrm{X}$ & $\mathrm{X}$ & $\mathrm{X}$ & \\
\hline AGHORA & $\mathrm{X}$ & $\mathrm{X}$ & & \\
\hline NXO & $\mathrm{X}$ & $\mathrm{X}$ & & \\
\hline
\end{tabular}

Table 3 - Test cases computed by CFD codes 


\section{RESULTS}

\section{COVO Test Case}

Many simulations were carried out by partners on this very simple test case and only the most representatives ones are presented in this paper at $M=0.5$. These results were originally presented in the beginning of 2015. The first objective was to compare the numerical accuracy and the associated cost of the different codes and on various grid densities and elements. The second objective was to mimic a stator/rotor/stator configuration with an intermediate sliding mesh.

On such a configuration, the analytical solution is known and so it is possible to measure accurately the numerical error. In that case various norms were tested, but all norm errors give more or less same tendency, and so only L2 norm is considered. To perform the analyses, 50 convective times were run and the L2 norm based on the analytical solution computed. To compare results in terms of CPU cost, the "Work Unit" reference proposed by Wang et al [35] is considered. A "Work Unit" is a reference CPU time scale to solve a basic problem defined and it was the only converged reference between partners to compare CPU efficiency of each code. In the end, for each code and each case, four simulations are carried out with four different grid densities and of course Work Units increase linearly with the grid densities.

The FE scheme TTGC is used for AVBP simulations while Aghora uses $\mathrm{p} 4$ scheme in 3D mode which could explain some CPU higher cost efficiency.

First, comparisons are presented in Figure 6 on regular quad grids. For all cases, 4 meshes are computed, with increasing Work Units as meshes are refined. The increase of order $\mathrm{p}$ of HO codes Jaguar and ARGO is clearly visible and is very beneficial in terms of CPU cost. Aghora is less efficient as it is run in 3D mode. The AVBP FV/FE code is more or less equivalent to $\mathrm{p} 2 \mathrm{HO}$ codes. In the end, Yales2 is not efficient in this quite high Mach number case. It appears that in that case $\mathrm{HO}$ codes with $\mathrm{p}>2$ are clearly the most efficient ones if we consider the ratio Accuracy / Work Units.

Same results with irregular quads are presented in Figure 7. The global conclusions are identical but we can clearly see that the advantage in HO CFD codes (DG or SD) is reinforced which could demonstrate that these codes are more resistant to bad grid quality. The results of HO codes are indeed slightly impacted by the grids and conversely FV codes are less accurate for the same Work Units.

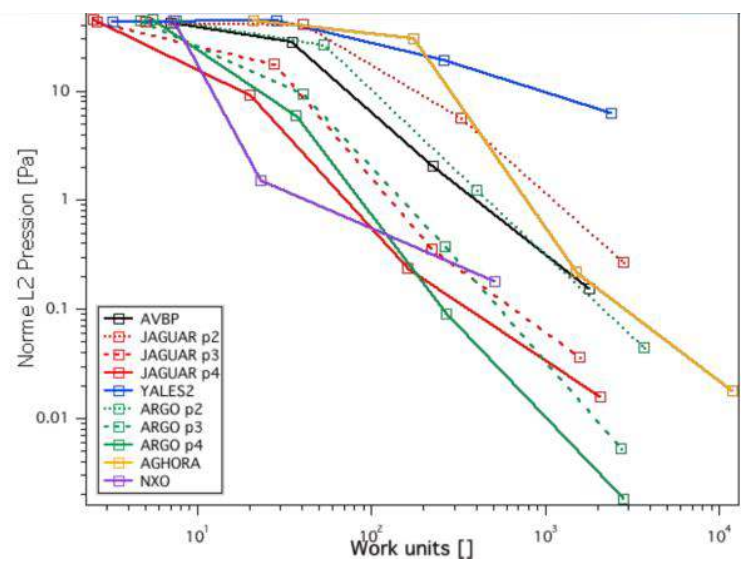

Figure 6 - L2 Norm on pressure of inviscid convection of a Vortex (without sliding mesh) $-M=0.5$ - Regular Quads

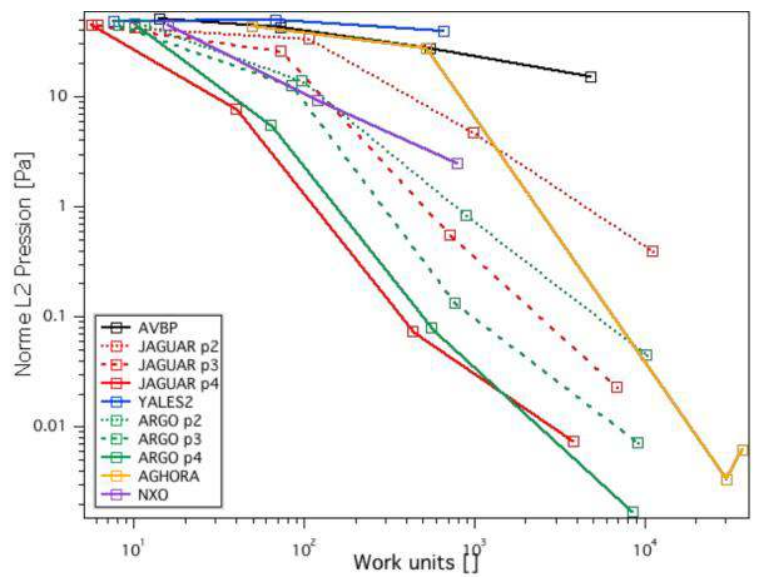

Figure 7 - L2 Norm on pressure of inviscid convection of a Vortex (without sliding mesh) $-M=0.5$ - Irregular Quads

To confirm previous observations, same comparisons on triangles at $M=0.5$ are plotted in Figure 8. The conclusion is identical: HO codes are not impacted and FV codes present degraded results.

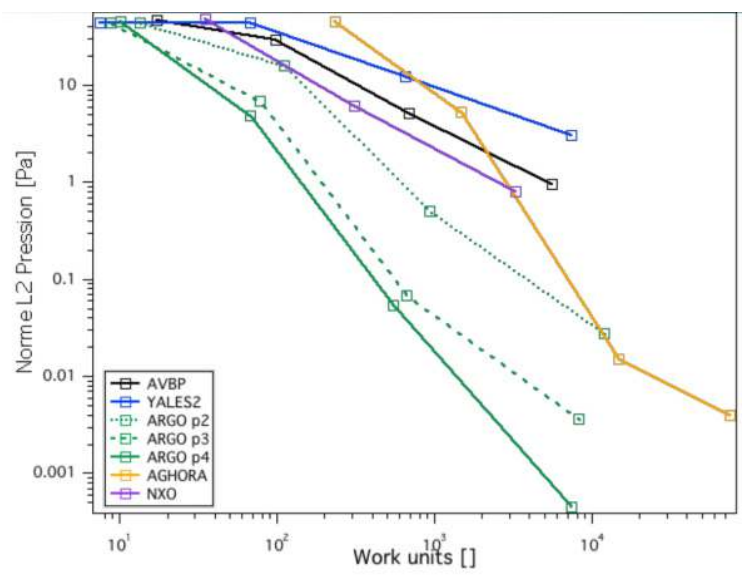

Figure 8 - L2 Norm on pressure of inviscid convection of a Vortex (without sliding mesh) $-M=0.5$ - Triangles 
Next a comparison of the Work Units achieved for a given accuracy based L2 norm on Pressure with a 1\% of accuracy compared to the analytical reference was performed and is presented in Table 4 for regular and irregular quads. The objective is to evaluate the ratio CPU Cost / Accuracy. Clearly, except for the research NXO code in some conditions, HO codes are the most efficient especially for $\mathrm{p} 4$ and $\mathrm{p} 3$.

\begin{tabular}{|l|r|}
\hline Code & Work Units \\
\hline NXO & 13.6 \\
\hline JAGUAR $\mathrm{p} 4$ & 23.0 \\
\hline ARGO $\mathrm{p} 4$ & 31.0 \\
\hline JAGUAR $\mathrm{p} 3$ & 44.8 \\
\hline ARGO P3 & 47.4 \\
\hline AVBP & 92.9 \\
\hline ARGO p2 & 127.9 \\
\hline JAGUAR $\mathrm{p} 2$ & 259.1 \\
\hline AGHORA & 326.7 \\
\hline YALES2 & 1887.9 \\
\hline
\end{tabular}

Table 4 - Work Units comparisons for a given accuracy on pressure (1\%) $M=0.5$ - Left: Regular quads - Right: Irregular quads

We now consider the same case but with the sliding block as presented in Figure 1. A first single test was performed with a translation velocity of $0 \mathrm{~m} / \mathrm{s}$. Of course, results should be very similar to the previous ones. In the end, since this conclusion was effectively obtained, these results are not presented. Considering now a translation speed of $50 \mathrm{~m} / \mathrm{s}$ and $300 \mathrm{~m} / \mathrm{s}$, presented in Figure 9, conclusions are very similar and we can observe that the translation speed has only a very limited impact on the accuracy and the cost of simulations. In the end, once more $\mathrm{HO}$ codes are less sensitive to grid quality than the FV codes. Considering HO codes the increase in $\mathrm{p}$ order allow a better efficiency to be obtained.

Note that the interface simulation cost represents more or less $30 \%$ of the total cost for AVBP code and less than $10 \%$ for $\mathrm{HO}$ codes because of the reduced required stencil. Some work demonstrates that an alternative strategy (storage) is feasible for FV code to drastically reduce this additional cost.

In conclusion of this fundamental test case, HO codes seem to be less expensive for a given accuracy, especially for high $\mathrm{p}$ order. Furthermore, the HO codes are clearly less sensitive to the grid quality since whatever the considered mesh elements, results remain more or less the same.
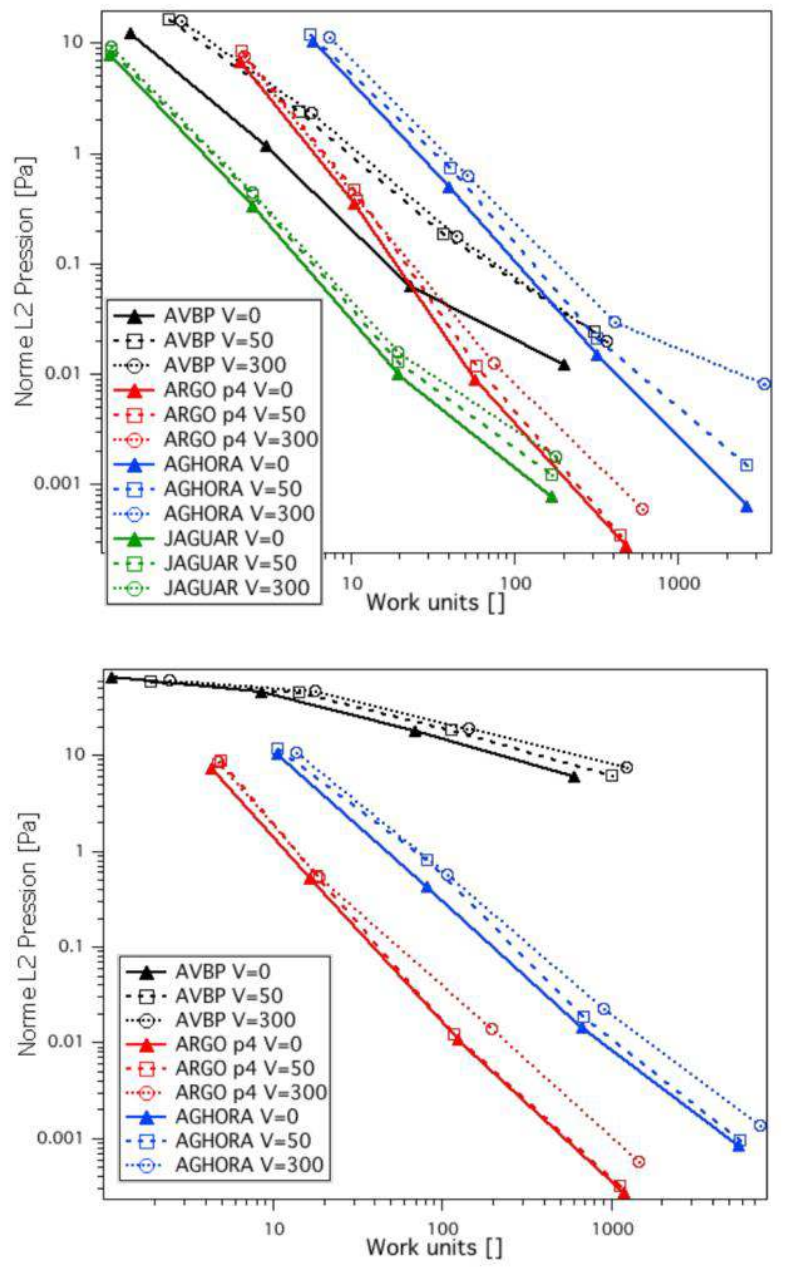

Figure 9 - L2 Norm on pressure of inviscid convection of a Vortex - Sliding mesh speed effect - $M=0.5$ - Regular

Quads: Top - Irregular Quads: Bottom

\section{Turbulent Channel}

The well-known turbulent heated periodic channel flow was computed by all partners and final results were compared at the beginning of 2015. The reference data is the DNS of Abe et al [1]. The forcing method is not presented in this paper. A reference grid was provided but partners were free to generate their own.

First, dimensionless velocity and temperature are compared to the reference DNS [1] in Figure 10 in various locations in the channel. All solutions match the reference data remarkably and only minor differences can be observed, almost negligible. 

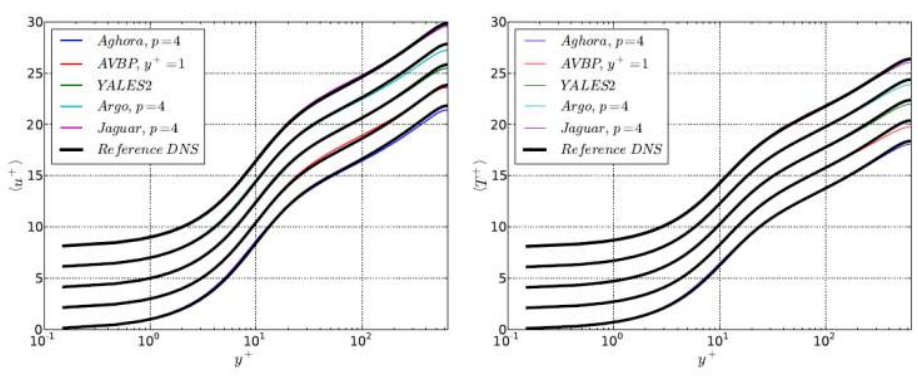

Figure 10 - Averaged dimensionless velocity and temperature for the turbulent plane channel

Averaged fluctuations of the same fields are presented in Figure 11 and even if differences are enhanced, all results are in a good agreement with the reference and it is almost impossible to discriminate solutions.
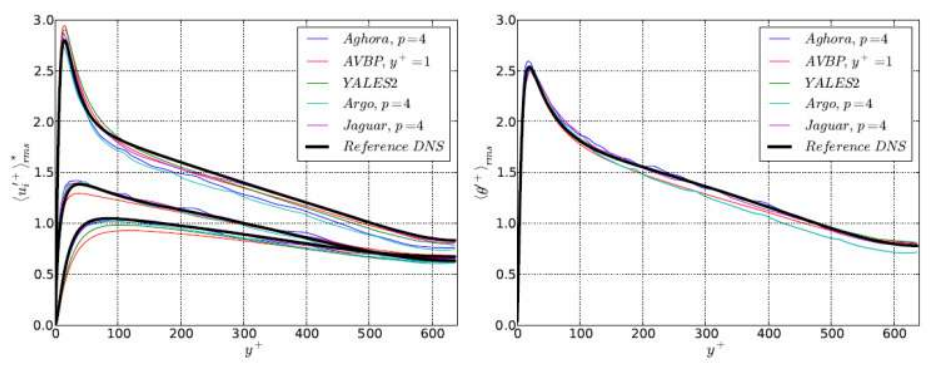

Figure 11 - Averaged dimensionless second order velocity and temperature for the turbulent plane channel

First discrepancies can be observed on Fourier analyses as presented in Figure 12 for the velocity and in Figure 13 for temperature at $y+=9.98$ (left) i.e. at the location of maximum fluctuations and $y+=298$ (right) i.e. at $\delta / 4$ from the wall. The sub-grid scale model and/or the filtering LES process is visible at higher frequencies. YALES2 FV code seems more dissipative than the others and AVBP FV/FE code and HO Jaguar and ARGO codes in a p4 mode are all in good agreement. Of course, contrary to the DNS reference, higher frequencies are not fully resolved in the LES simulations.
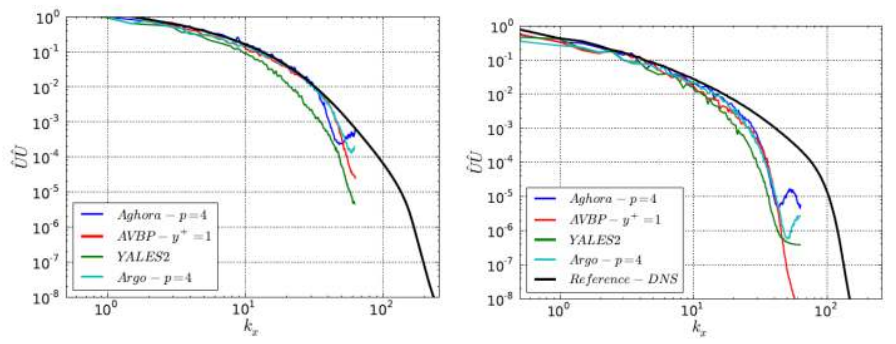

Figure 12 - Spectrum of axial velocity $y+=9.98$ (maximum fluctuation location) left $/ y+=298.20(\delta / 4)$ right
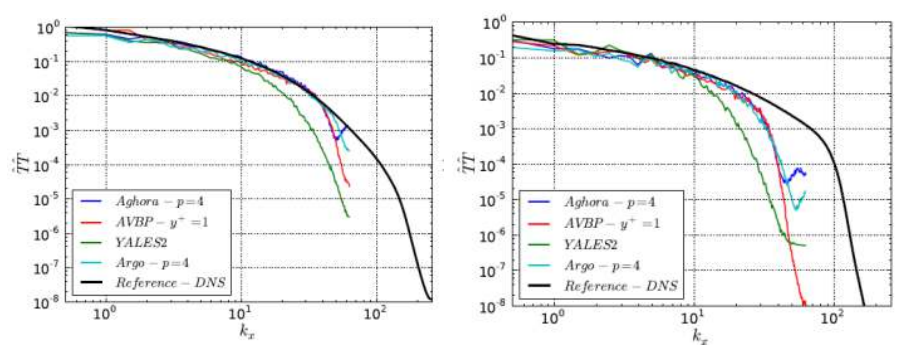

Figure 13 - Spectrum of temperature $y+=9.98$ (maximum fluctuation location) left $/ y+=298.20$ (S/4) right

To conclude on the accuracy of the different results, no clear discrepancies between codes can be observed and all are very accurate compared to the reference DNS result. It appears that this test case may be "too easy" for partners and in the end, the only way to obtain differences between codes is to compare their CPU cost as presented in Figure 14. In this figure, all CFD codes are more or less in the same range of efficiency except YALES2 which is an incompressible code and is 5 times faster than the others on this test case at this moment.

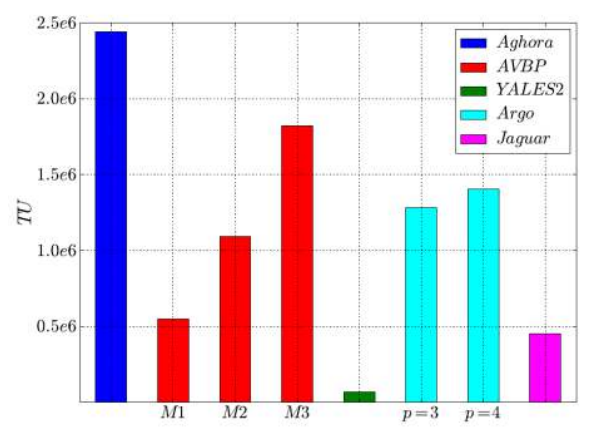

Figure 14 - Total CPU time for the turbulent plane channel

\section{LS89 Turbine Cascade}

Continuing in increasing test case complexity, we move on to the LS89 turbine vane (Arts et al [2]). The case adds three key features: boundary layer transition, shocks and vortex shedding. Results from YALES2, AVBP, JAGUAR and ARGO, alongside a RANS reference computed with the elsA software [3] by Safran with a Menter SST model and a $\gamma-\operatorname{Re}_{\theta}$ transition model from Menter-Langtry will be compared.

Let us first look at the MUR129 case (See [2]). This case has a $1 \%$ inlet turbulence intensity, no shocks and mostly laminar boundary layers. Transition appears only on the suction side, very close to the trailing edge. This phenomenon can be observed on Figure 15, through the sharp rise in heat transfers. On this figure, negative curvilinear abscissa corresponds to the pressure side, $s=0$ to the leading edge, and positive curvilinear abscissa to the suction side. 


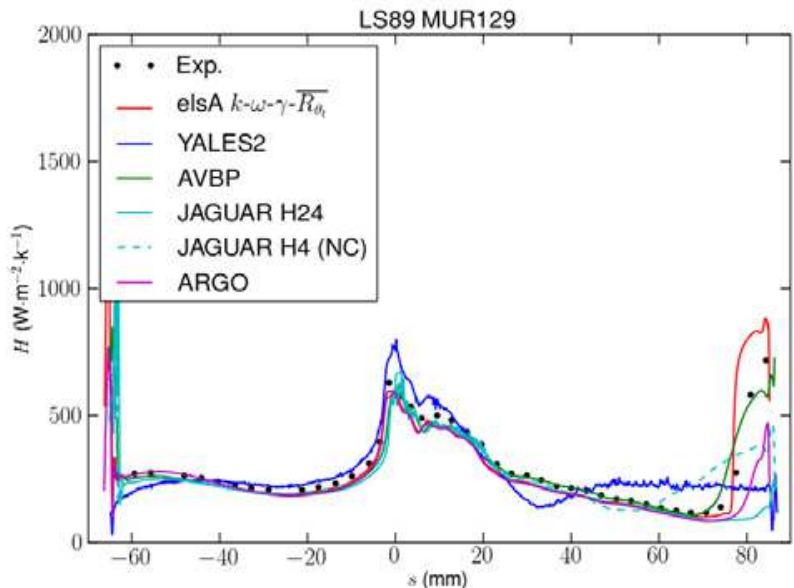

Figure 15 - Heat Transfer as a function of curvilinear abscissa for the MUR129 case

In Figure 15, we can see that all codes do predict a laminar boundary layer on the pressure side. There are however significant differences on the suction side. For instance, the YALES2 code displays a very early transition. This is due to the impact on the suction side of high acoustic levels emanating from the trailing edge vortex shedding of the next blade. This problem was since solved by simply refining the mesh. The JAGUAR computation on the coarse mesh (H24) does not present any laminar-to-turbulent transition. Transition seems to appear on the finer mesh (H4), but as the computation was not converged by the time results were due, it is hard to conclude. Computations by these two codes show how sensitive transition is to the refinement level. As for ARGO (p3) and AVBP, both computations experience boundary layer transition at the correct location, yielding to good results overall.

Let us now move on to the MUR235 computations (See [2]). This case adds the complexity of having a higher inlet turbulence intensity of $5 \%$. Furthermore, the decay of turbulence from the inlet to the leading edge must match the experiments in order to have the correct boundary layer transition. Every partner was free to use its own turbulence generation technique. Note that synthetic turbulence generation is still an important challenge for LES simulations. During the experimental campaign in VKI [2], the $T u$ decay upstream of the leading edge of the blade was characterized as well as the integral length scale estimated. These parameters were imposed into simulations but we have to note that the integral length scale is higher than the computed span domain limiting the accuracy of such simulations.

We can see on Figure 16 how complicated it is difficult to recover the heat flux profile with an LES code. Depending on the code and turbulence injection technique, very different turbulence intensities at the leading edge and turbulence decays are obtained. It is worth explaining at this time that the AVBP computations were run with two injection techniques synthetic turbulence injection and use of a precursor simulation - and two inlet turbulence intensities - $6 \%$ and $18 \%$ [28]. Note that the RANS result obtain with the elsA software, modeled turbulence injected and the transition modeling perfectly match the experiments. This result was easily obtained which proves that inlet turbulence injection is easier with RANS (modeled) that with LES (resolved).

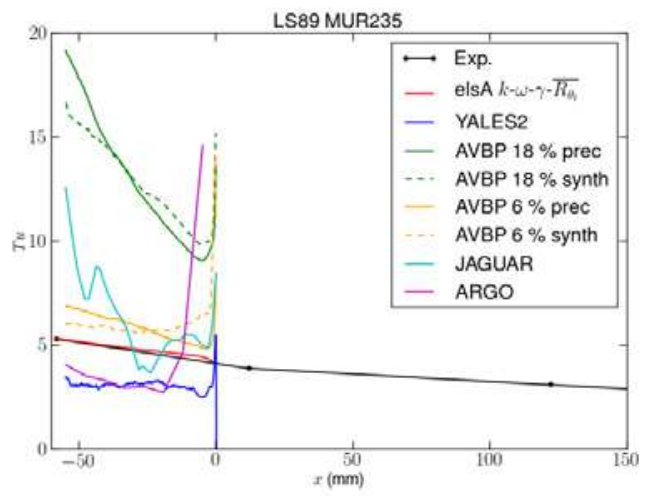

Figure 16-Turbulent decay upstream of the leading edgeMUR235

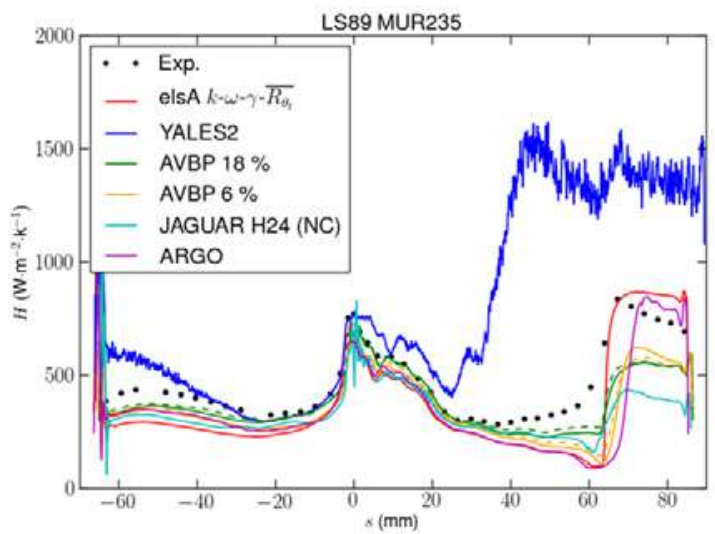

Figure 17 - Heat Transfer for the MUR235 case

On the MUR235 case, the boundary layer on the pressure side is stable and does not experience transition. However, due to the high freestream turbulence intensity, some turbulence structures are present in the boundary layer, which slightly raises the heat fluxes in that area compared to MUR129. The RANS computation gives a fully laminar solution and therefore does not capture this slight rise in heat fluxes. This phenomena is however captured by the LES computations, though the heat fluxes are still under predicted. The YALES2 calculation experiences transition, which is inaccurate, mostly because of numerical instabilities. 
The suction side of the MUR235 is extremely difficult to predict. The boundary layer is stable until $s=20 \mathrm{~mm}$, where turbulent spots start appearing. However the boundary layer is fully turbulent only around $s=60 \mathrm{~mm}$. On the spanwise and time-averaged experiments, this creates a plateau of intermediate levels of heat fluxes. The averaging does not allow us to discriminate between the presence of high intensity but intermittent structures, or high intensity but localized in span structures, or low intensity and uniform in both space and time structures.

Looking at Figure 17, we can see that none of the LES computations with the correct inlet turbulence level can capture even a trend towards this plateau (except maybe the JAGUAR computation, but the simulation is not converged). The AVBP simulations start capturing that plateau, but only after raising the inlet turbulence intensity to $18 \%$. We can also see on Figure 21 that the ARGO simulations do predict some turbulent structures in that regions, though they do not show on the heat fluxes.

Looking at Figure 18 to Figure 21, we can see that macroscopically, the results are very different. In particular, the AVBP $6 \%$ and ARGO simulations give the same time and span averaged results, but the transition process is different. In the AVBP simulation (Figure 20), the Klebanoff modes present until the suction peak tend to disappear, leaving only very fine turbulent structures in the laminar boundary layer. The boundary layer transitions due to the impact of the shock. By opposition, in the ARGO simulation (Figure 21), the Klebanoff modes decay into turbulent spots. These spots are intermittent and do not seem to form a fully turbulent boundary layer. The boundary layer transitions later, apparently through a bypass process.

We can also see that the four simulations give very different wakes. The AVBP simulation has a very coherent 2D wake, the JAGUAR simulation also has a marked von Karman street but with many 3D structures around it, while the YALES2 and ARGO simulations have a fully $3 \mathrm{D}$ wake with an almost indiscernible von Karman street.

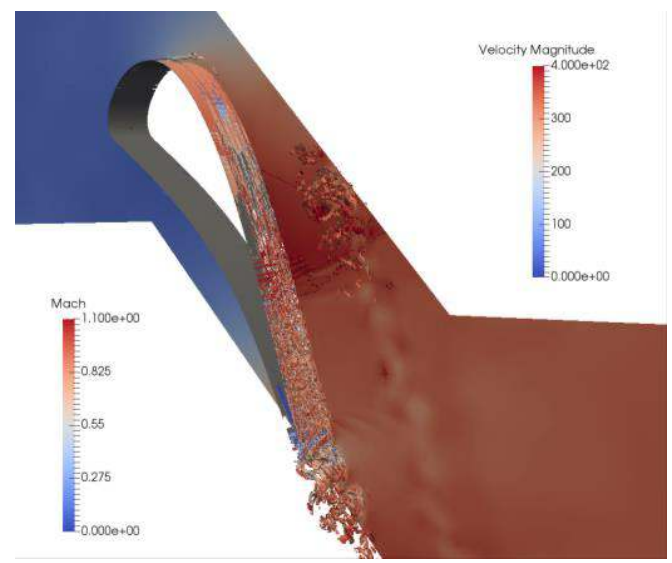

Figure 18 - iso $10^{10} Q$-Criterion colored by velocity magnitude and slice colored by Mach number - Jaguar Code-

\section{MUR235}

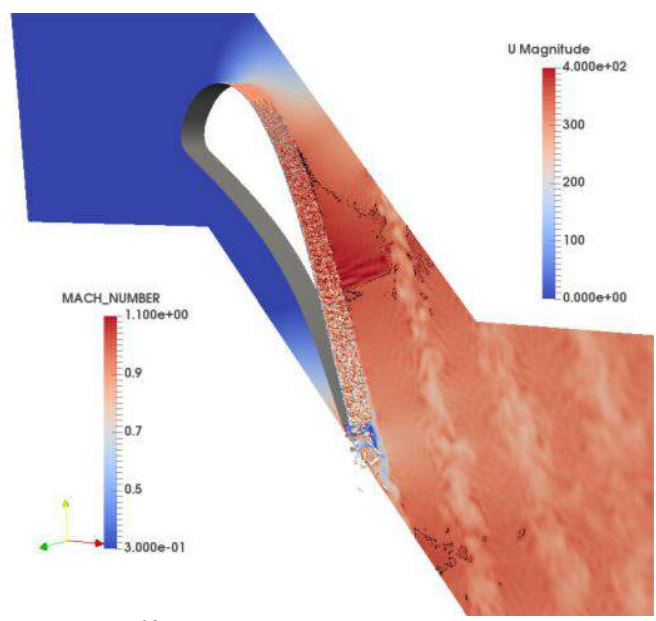

Figure 19 - iso $10^{10}$ Q-Criterion colored by velocity magnitude and slice colored by Mach number - YALES2 Code - MUR235

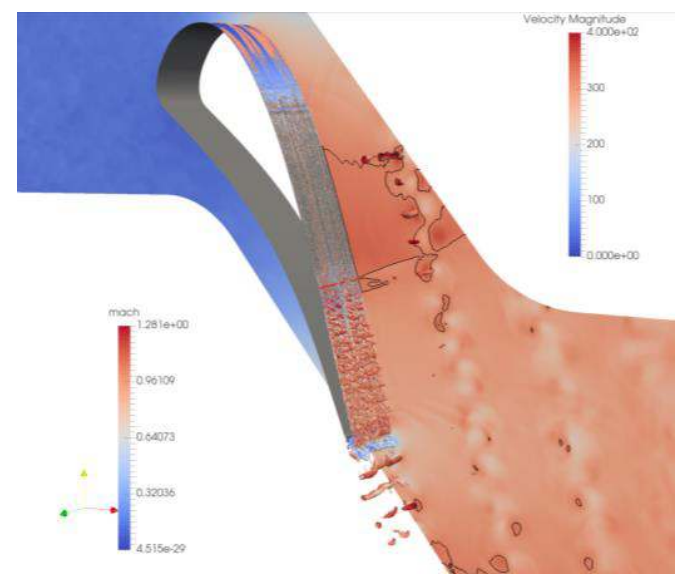

Figure 20 - iso $10^{10} Q$-Criterion colored by velocity magnitude and slice colored by Mach number-AVBP CodeMUR235 


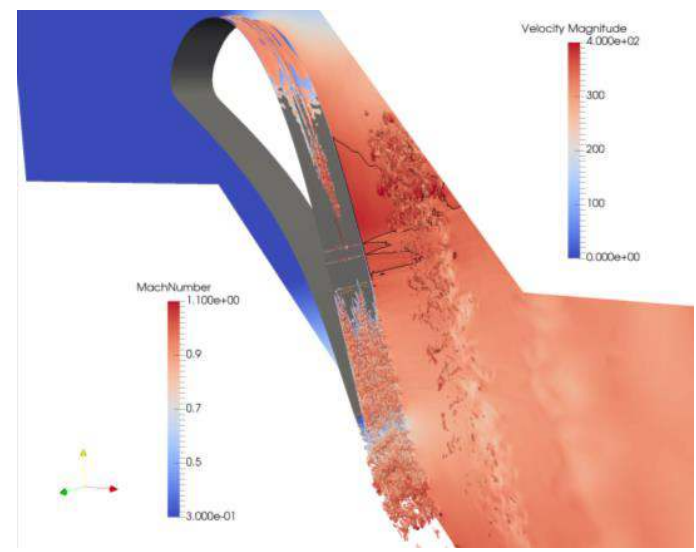

Figure 21 - iso $10^{10} Q$-Criterion colored by velocity magnitude and slice colored by Mach number - Argo CodeMUR235

The LS89 High Speed (HS) is a mock test case created for the CN2020 project. It is the same as the MUR235, except that the outlet pressure was significantly lowered in order to create intense shocks. This case was only computed by the ARGO and AVBP team (and by Safran for the RANS simulation).

In this case, the fish tail shock impacts the blade, where it is reflected and then impacts the wake. In the RANS simulation (Figure 22), the wake is too diffused to cause any reflection. In opposition, the AVBP wake (Figure 23) is very coherent and causes a single, large reflection. In the ARGO simulation (Figure 24), the wake has much finer and more 3D structures. The shock diffuses downstream quite quickly because of the sponge layer. This creates complex weak shocks and reflection patterns between the wake and the blade.

The LS89 test cases show that the LES add physical phenomenon compared to the RANS simulations, but the added physics differ greatly depending on the chosen methods and meshes. They also show that boundary layer transition remains very difficult to predict. It is highly sensitive to the turbulence generation method, as well as its advection. Free stream grids tends to be too coarse to properly advect turbulence, leading to discrepancies in the transition zones. Another key finding of this project is the differences in the vortex shedding of the different methods. This is particularly disconcerting, as this is specifically a phenomenon we expect to better capture through LES.

The interested reader is referred to [28],[29] for more details on the AVBP simulations.

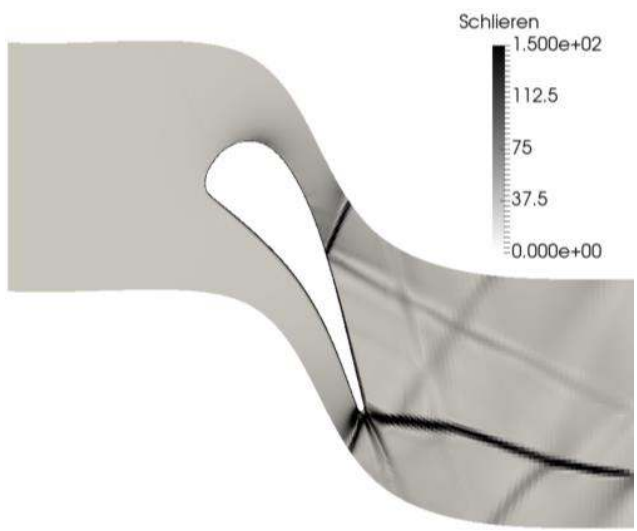

Figure 22 - Schlieren flow visualization - elsA CodeLS89 HS - Steady RANS

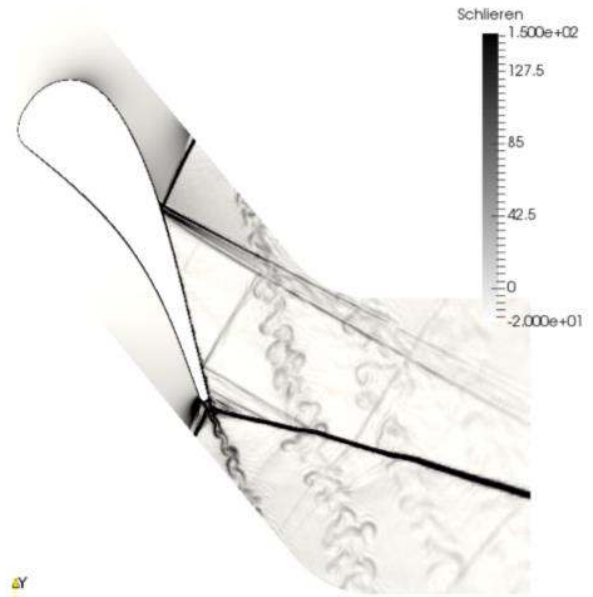

Figure 23 - Schlieren flow visualization - AVBP CodeLS89 HS

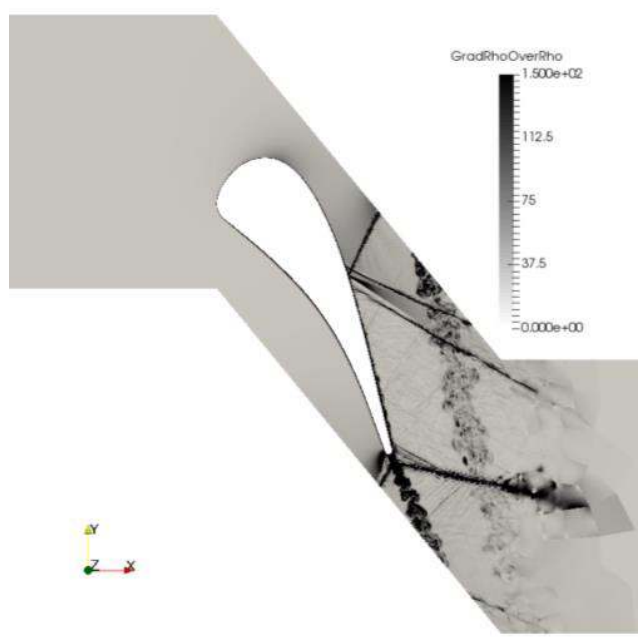

Figure 24-Schlieren flow visualization - ARGO Code - LS89 $H S$ 


\section{Multistage CREATE2Bis}

This simulation had to be carried out by Cenaero with the ARGO code and Cerfacs with the TurboAVBP code but, mostly because of the lack of CPU available it could not be performed by Cenaero. The grid (Figure 25) was generated for a highorder CFD DG code from a classical grid made with the Autogrid Software from NUMECA through a coarsening procedure which allows high order elements to be created.

Regarding the TurboAVBP simulation, fully unstructured grids of successive refinements were generated with Centaur Software. Their main characteristics are gathered in Table 5 . With more than 1 billion of DoFs, M3 grid fulfills the project requirement. M2 and M3 grids consist of tetrahedra cells away from blade walls and of prisms layers on blade walls to allow for a proper wall refinement while limiting the amount of cells. The maximal $\mathrm{y}^{+}$value obtained for $\mathrm{M} 2$ and $\mathrm{M} 3$ allows for a relevant use of a wall law, whereas this value appears too large on M1 to expect proper near-wall flow physics.

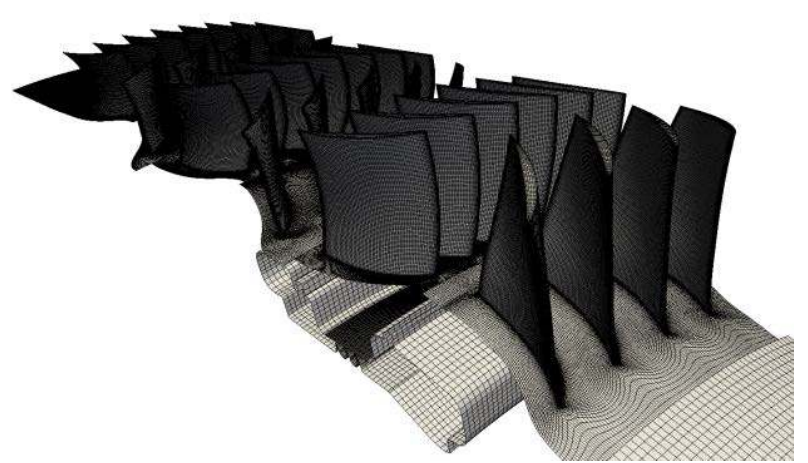

Figure 25 - Grid for DG simulation on the CREATE2bis compressor for the ARGO Code.

\begin{tabular}{|lccc|}
\hline & M1 & M2 & M3 \\
\hline Nb. cells total $\left(\times 10^{6}\right)$ & 88 & 194 & 1032 \\
\hline Nb. prisms layers on blades & 0 & 3 & 10 \\
\hline Nb. cells in rotor tip clearances & 3 & 6 & 20 \\
\hline $\mathrm{y}^{+}$max. blades & $\approx 500$ & 110 & 40 \\
\hline
\end{tabular}

Table 5 - Mesh characteristics for the AVBP CFD code on the Create2Bis compressor

The numerical parameters of the simulations are detailed in [13],[14]. The CPU cost, directly related to the mesh size and the time step, increases from one order of magnitude from M1 to M2 and M2 to M3, as shown in Table 6. For M1 and M2, CPU costs are affordable and similar to current simulations that can be performed in industry. Thus several revolutions and operating points could be simulated up to convergence. Because of its large CPU cost, only 2 revolutions could be performed for M3 grid, starting from a converged M2 solution. Mass flow convergence could not be strictly reached, its relative evolution being less than $1 \%$ during the last revolution.

TurboAVBP simulations of CREATE have been successfully ported on different HPC architectures from GENCI (OCCIGEN/CINES，TURING/IDRIS，COBALT/CCRT) and Meteo-France (BEAUFIX, PROLIX), up to more than 100000 CPU cores. Hence initial CN2020 objectives are fulfilled since the capability of TurboAVBP has been demonstrated on this challenging test case.

\begin{tabular}{|l|c|c|c|}
\hline & M1 & M2 & M3 \\
\hline dt (s) & $4 \mathrm{e}-8$ & $2 \mathrm{e}-8$ & $5.4 \mathrm{e}-9$ \\
\hline nb it./rev. $\left(\times 10^{3}\right)$ & 130 & 260 & 963 \\
\hline CPU time/rev. $\left(\times 10^{3} \mathrm{~h}\right)$ * & 17 & 165 & 2020 \\
\hline
\end{tabular}

Table 6 - CPU cost for the CREATE2bis configuration (*on Haswell 2.6 GHz cores)

Going beyond HPC evaluation, some comparisons with available experimental data have been performed [13],[14], and the main outcomes are recalled here. The turbulent structures simulated by LES are shown in Figure 26 (a) \& (b). The complex flow taking place in the rotor tip clearances are specifically highlighted (tip leakage vortices, tip separation vortices). The effect of grid refinement is clearly visible when comparing Figure 26 (a) \& (b), as shown by much finer turbulent structures captured with M3 grid. Rotor tip vortices are known to significantly contribute to the overall aerodynamic losses of the compressor, thus the need for their accurate prediction.

Aerodynamic losses are represented in Figure 27 (a) and (b) as entropy fields at $50 \%$ and $95 \%$ of the duct height $(h / H)$. At $50 \% h / H$, complex interactions of upstream wakes with downstream blades are visible for each row. These interactions are responsible for losses that progressively increase through the successive bladed rows. Close to the tip casing (Figure 27 (b)), losses are much higher in the rotors. Indeed they are caused by the complex tip vortices observed in Figure 26 (b).

Measured iso-speed characteristic curves of CREATE2bis compressor at $11543 \mathrm{rpm}$ are shown in Figure 28 and Figure 29 in terms of total pressure ratio and isentropic efficiency respectively. For comparison purposes, special attention has been paid to post-process LES (see [19] for details). As mentioned earlier, several operating points could be simulated with M1 and M2, from close to choke to close to surge conditions. The shapes of M1 iso-speed lines are satisfactory with respect to measurements, however the efficiency is overpredicted, and this grid is not able to reach the last stable point at off-design conditions. On the contrary, M2 grid correctly predicts the performances of the compressor, in terms of 
pressure ratio and efficiency (within the margin error). Note the important margin error $3 \%$ caused by complex inlet measurements. Moreover this refinement level allows to closely approach the last stable experimental operating point identified as off-design conditions. This suggests that wall-modeled LES with intermediate refinement could be a viable approach for performance prediction at off-design conditions [14] for which low order techniques often fail to provide correct results. For M3, statistics have been recorded during the last revolution and corresponding points are shown as green triangles. The overestimation of mass flow may be the consequence of the lack of convergence.
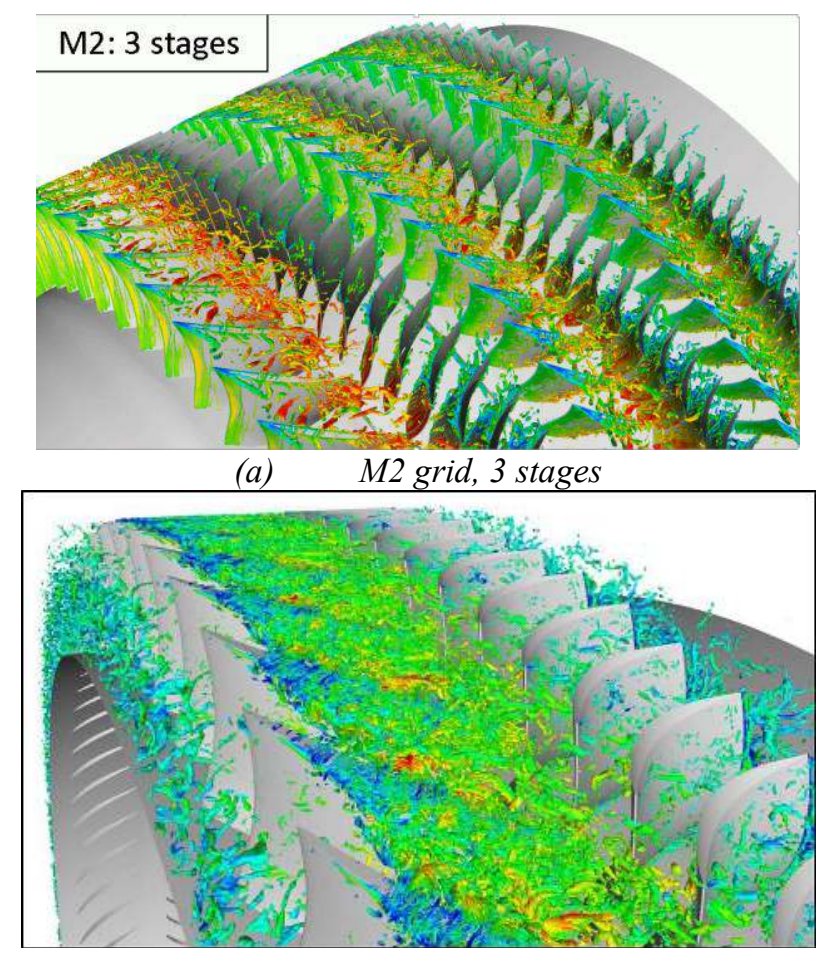

(b) M3 grid, last stage

Figure 26 - Instantaneous flow visualization of the turbulent structures - Iso-Q criteria colored by $M$

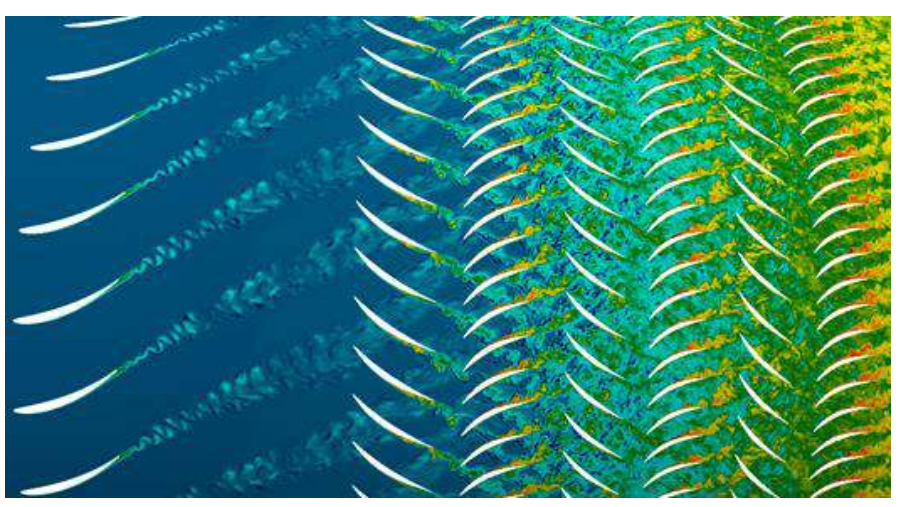

(a) $-h / H=50 \%$

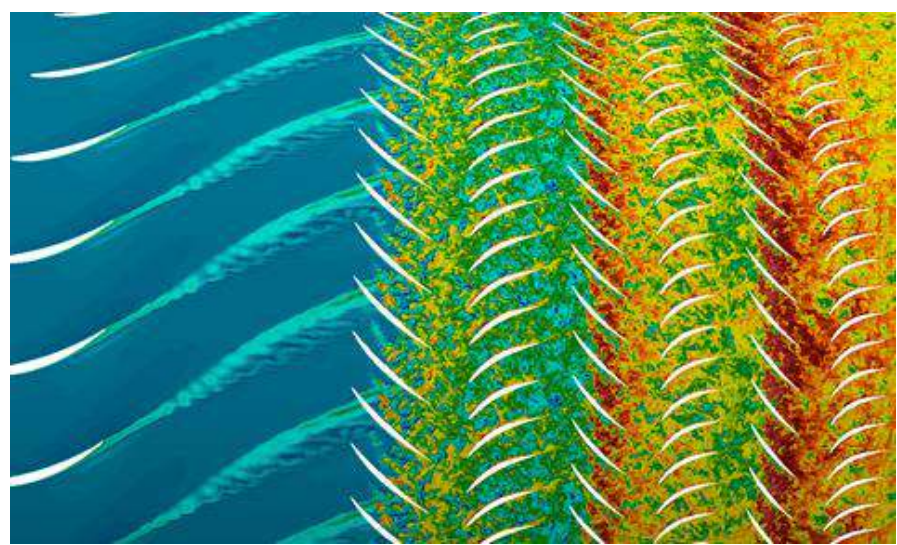

(b) $-h / H=95 \%$

Figure 27 - Instantaneous entropy visualization - M3 grid same color scale

Figure 30 represents radial profiles of total pressure and total temperature averaged in time and in the azimuthal direction in section 270 (see Figure 4). The experimental data corresponds to the operating point VA39 in Figure 28. These comparisons show the benefit of using a properly refined LES grid such as M3 to accurately predict the mean flow along the entire duct height. Conclusions are similar regarding the unsteady flow, correctly predicted by grid M3 as shown in Figure 31 by turbulence intensity radial profiles downstream rotors 2 and 3 . Note that close to the tip, the turbulence intensity is underestimated by all simulations, most refined ones being the closest but a clear deficit is still observed.

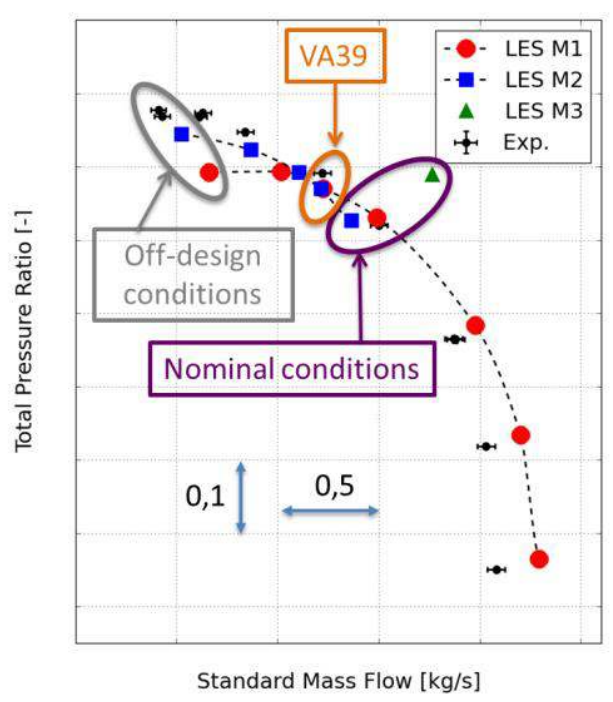

Figure 28 - Characteristic of the CREATE2bis compressorPressure Ratio 


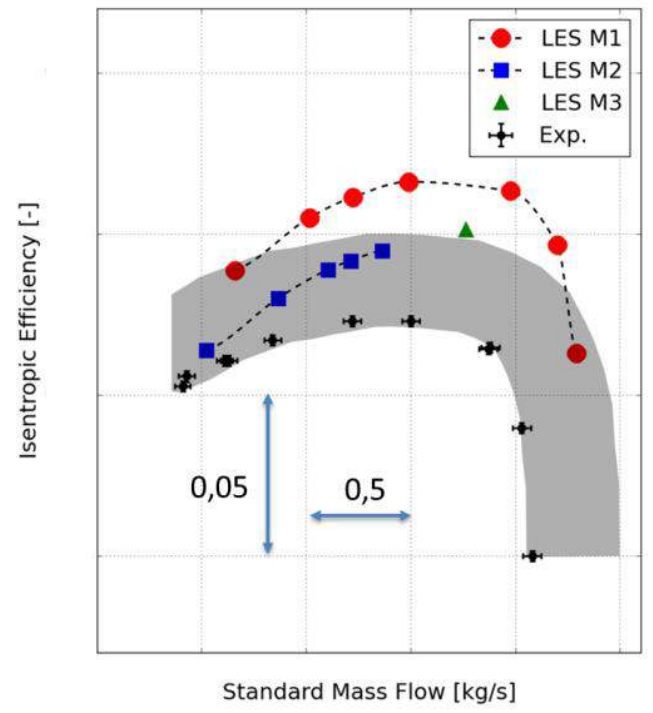

Figure 29-Characteristic of the CREATE2bis compressor Isentropic efficiency (3\% margin error in grey)
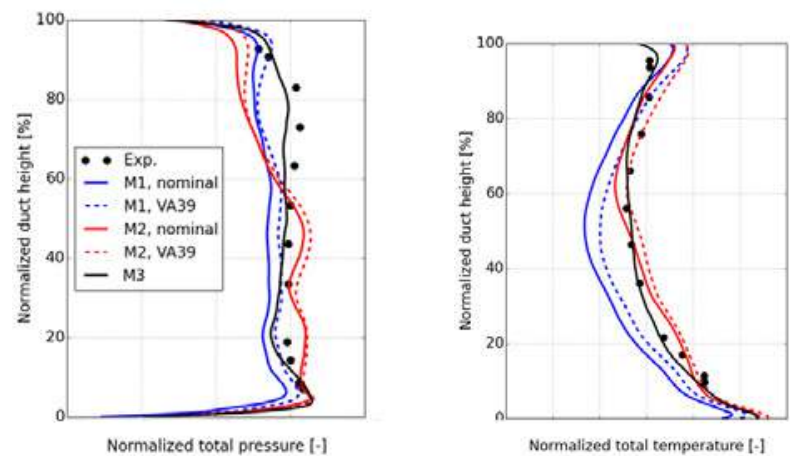

Figure 30 - Normalized total pressure and temperature in a section downstream of the S1
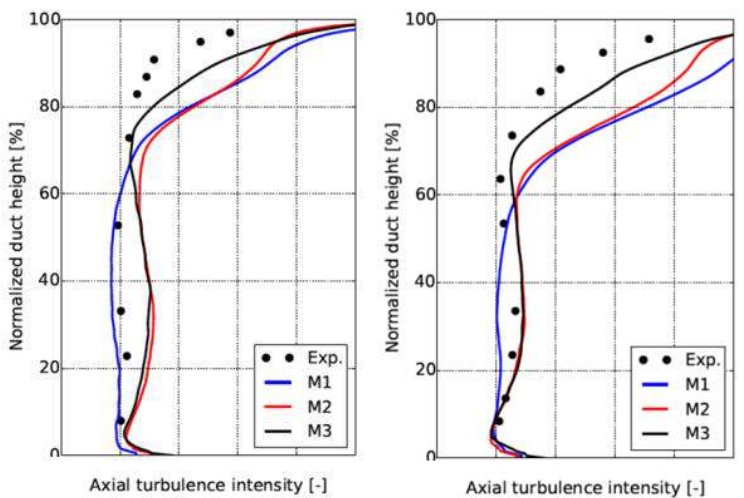

Figure 31 - Turbulence intensity downstream of Rotor 2 (left) and Rotor 3 (right) [14].

For more analysis of these results, please refer to [13] and

\section{CONCLUSIONS AND PERSPECTIVES}

This paper compares different CFD codes and numerical approaches for LES simulations on various tests cases starting from a very fundamental one up to a complex multistage axial compressor. The objective was to select the best method for future LES simulations of turbomachinery. In the end, FV codes are of course more mature than $\mathrm{HO}$ ones, but CPU efficiency versus accuracy is in the same order of magnitude. $\mathrm{HO}$ codes are more efficient with $\mathrm{p} 4$ or $\mathrm{p} 3$ orders. It seems that $\mathrm{HO}$ codes are more robust to bad grid quality than FV codes.

Lots of work remain on synthetic turbulence generation, CPU efficiency, laminar-to-turbulent transition, grid generation for all codes and methods... before a daily use for conception.

The best way in increase accuracy and CPU costs are, from author's point of views, to work and automatic grid (and order) adaptation to limit numerical errors and to develop efficient and accurate wall functions to drastically reduce DoFs numbers and consequently CPU cost. Note that high order grid generation for HO codes is a strong necessary research topic too.

\section{ACKNOWLEDGMENTS}

The authors gratefully acknowledge scientific committee members of the project: Pierre Leca, Bruno Audebert and Denis Ricot,

Special thanks to Thomas Fédérici who originally managed the project.

\section{REFERENCES}

[1] H. Abe, H. Kawamura and Y. Matsuo, "Surface heat-flux fluctuations in a turbulent channel flow up to $\mathrm{Re}=1020$ with $\operatorname{Pr}=0.025$ and 0.71 ", International Journal of Heat and Fluid Flow, vol. 25, pp. 404|419, 2004.

[2] T. Arts, M. de Rouvroit, Lambert, and A.W. Rutherford. "Technical report", Von Karman Institute for Fluid Dynamics, 1990.

[3] L. Cambier, S. Heib, and S. Plot. The Onera elsA CFD software: input from research and feedback from industry. In: Mechanics \& Industry 14.3 (2013), pp. 159-174.

[4] C. Carton de Wiart, K. Hillewaert, M. Duponcheel, and G. Winckelmans, "Assessment of a discontinuous Galerkin method for the simulation of vortical flows at high Reynolds number", Int. J. Numer. Meth. Fluids, 74:469493, 2014.

[5] C. Carton de Wiart, C., K. Hillewaert, L. Bricteux, and G. Winckelmans, "Implicit LES of free and wall bounded turbulent flows based on the discontinuous Galerkin/symmetric interior penalty method". Int. J. Numer. Meth. Fluids, 78, 2015.

[6] A. Cassagne, J-F. Boussuge, G. Puigt, N. Villedieu, A. Genot, JAGUAR: a new CFD code dedicated to massively parallel high--order LES computations on complex 
geometry, 50th 3AF International Conference on Applied Aerodynamics (AERO 2015), 30 March-1 April, 2015.

[7] D. Chapman. "Computational aerodynamics development and outlook". In: AIAA J 17.12 (1979), pp. 1293-1313.

[8] H. Choi and P. Moin. "Grid-point requirements for large eddy simulation: Chapman's estimates revisited". In: Physics of fluids 24.1 (2012), p. 011702.

[9] A.J. Chorin, "Numerical solution of the Navier-Stokes equations", Math. Comput., 1968, 22(104), 745-762

[10]O. Colin and M. Rudgyard. Development of high-order Taylor-Galerkin schemes for unsteady calculations. J. Comput. Phys. , 162(2):338-371, 2000.

[11] M. de la Llave Plata, V. Couaillier, and M.C. le Pape. "On the use of a high-order discontinuous Galerkin method for DNS and LES of wall-bounded turbulence." Computers \& Fluids, 2017.

[12] M. de la Llave Plata, E. Lamballais, and V. Couaillier. «A discontinuous Galerkin variational multiscale approach to LES of turbulent flows." In Proc. of the ERCOFTAC Workshop Direct and Large-Eddy Simulation 11 (DLES11), (to appear), 2017.

[13] J. de Laborderie, F. Duchaine, O. Vermorel, L. Gicquel, S. Moreau, "Application of an Overset Grid Method to the Large-Eddy Simulation of a High-Speed Multistage Axial Compressor", ASME TurboExpo 2016, GT2016-56344

[14] J. de Laborderie, F. Duchaine, L. Gicquel, "Analysis of a high-pressure multistage axial compressor at off-design conditions with coarse Large Eddy Simulations", 12th European Turbomachinery Conference, 2017, ETC2017125

[15] Duchaine, F., Jauré, S., Poitou, D., Quémerais, E., Staffelbach, G., Morel, T. and Gicquel, L. Y. M. (2015) Analysis of High Performance Conjugate Heat Transfer with OpenPALM coupler, Computational Science and Discovery, 8 (1), pp. 15003.

[16] A. Frère, C. Carton de Wiart, K. Hillewaert, P. Chatelain, and G. Winckelmans. "Application of wall-models to discontinuous Galerkin LES”, Phys. Fluids 29, 2017.

[17]N. Gourdain et al. "Large eddy simulation of flows in industrial compressors: a path from 2015 to 2035". In: Phil. Trans. R. Soc. A 372.2022 (2014), p. 20130323.

[18]K. Hillewaert, "Development of the Discontinuous Galerkin Method for high-resolution, large scale CFD and acoustics in industrial geometries". $\mathrm{PhD}$ thesis, Ecole polytechnique de Louvain/iMMC, 2013

[19] K. Hillewaert, J.S. Cagnone, S.M. Murman, A.Garai, Y. Lv and M. Ihme, "Assessment of high-order DG methods for LES of compressible flows", Center for Turbulence Research, Stanford, Proceeding of the summer program 2016.

[20] J. Kim, P. Moin, "Application of a fractional-step method to incompressible Navier-Stokes equations", J. Comput. Phys., 1985, 59(2), 308-323
[21] T.H. Lê, J.-M. Le Gouez, E. Garnier, "High accuracy flow simulations: Advances and challenges for future needs in aeronautics", Computers \& Fluids 43 (2011) 90-97

[22] J.-M. Le Gouez, V. Couaillier, F. Renac, "Numerical Properties and GPU implementation of a High Order Finite Volume Scheme", 48th AIAA Aerospace Sciences Meeting, Orlando, FLORIDE, USA, January 2010.

[23] M. Malandain, N. Maheu, V. Moureau, "Optimization of the deflated conjugate gradient algorithm for the solving of elliptic equations on massively parallel machines", J. Comp. Physics, 2013, 238, 32-47

[24] V. Moureau, C. Bérat, H. Pitsch, "An efficient semiimplicit compressible solver for large-eddy simulations", J. Comp. Physics 226, 2007, 1256-1270

[25]F. Renac, M. de la Llave Plata, E. Martin, J.-B. Chapelier, and C. Couaillier. "Aghora: A High-Order DG Solver for Turbulent Flow Simulations, IDIHOM: Industrialisation of High-Order Methods - A Top Down Approach." Notes on Numerical Fluid Mechanics and Multidisciplinary Design, 128, 2015.

[26]F. Renac, "Stationary Discrete Shock Profiles for Scalar Conservation Laws with a Discontinuous Galerkin Method", SIAM Journal on Numerical Analysis Vol. 53, No. 4, pp. 1690-1715

[27] T. Schoenfeld and M. Rudgyard. Steady and unsteady flow simulations using the hybrid flow solver avbp. AIAA Journal , 37:1378-1385, 1999. 25, 27

[28] L. Segui and L. Gicquel and F. Duchaine and J. de Laborderie, "LES of the LS89 cascade: influence of inflow turbulence on the flow predictions", 12th European Turbomachinery Conference, 2017, ETC2017-159

[29] Segui, L., Gicquel, L., Duchaine, F., \& de Laborderie, J. (2018). «Importance of boundary layer transition in a high-pressure turbine cascade using LES". Submitted to ASME TurboExpo 2018.

[30] Slotnick, J.; Khodadoust, A.; Alonso, J.; Darmofal, D.; Gropp, W.; Lurie, E. \& Mavriplis, D. , "CFD Vision 2030 Study: A Path to Revolutionary Computational Aerosciences", NASA, 2014, NASA/CR-2014-218178

[31] Spalart, P. and Jou, W. and Strelets M. and Allmaras, S., "Comments on the Feasibility of LES for Wings and on a Hybrid RANS/LES approach", In: Proceedings of the 1st AFSOR Int. Conf. on DES/LES, pp 137-147, 1997.

[32] Tucker, P.G., "Computation of unsteady turbomachinery flows: Part 1 - Progress and challenges", Progress in Aerospace Sciences, vol. 47, pp 522-545, 2011

[33] Tucker, P.G., "Computation of unsteady turbomachinery flows: Part 2 - LES and hybrids", Progress in Aerospace Sciences, vol. 47, pp 546-569, 2011

[34] J. Vanharen, G. Puigt, X. Vasseur, J-F. Boussuge, P. Sagaut, Revisiting the spectral analysis for high-order spectral discontinuous methods, Journal of Computational Physics, 337, pp. 379-402, 2017. 
[35]Z.J. Wang, K. Fidkowski, R. Abgrall, F. Bassi, D. Caraeni, A. Cary, H. Deconinck, R. Hartmann, K. Hillewaert, H.T. Huynh, N. Kroll, G. May, P.O. Persson, B. van Leer, M. Visbal, "High-Order CFd Methods: Current Status and Perspective”, Int. J. Numer. Meth. Fluids, 2012; 00:1-42
[36] Wang, G., Duchaine, F., Papadogiannis, D., Duran, I., Moreau, S., \& Gicquel, L. Y. (2014). An overset grid method for large eddy simulation of turbomachinery stages. Journal of Computational Physics, 274, 333-355. 